\title{
String fluid dynamics in general relativity
}

\author{
Larry L Smalley† and Jean P Krisch $\ddagger$ \\ $\dagger$ Department of Physics, University of Alabama in Huntsville, Huntsville, AL 35899, USA \\ $\ddagger$ Department of Physics, University of Michigan, Ann Arbor, MI 48109, USA \\ Received 21 January 1997, in final form 8 May 1997
}

\begin{abstract}
The general, energy-momentum tensor for a dynamical, string fluid in general relativity is presented using the Ray-Hilbert variational principle. The calculations are given for both the standard and the extended thermodynamics versions in which the latter includes the string as thermodynamic variables. In general relativity, it is shown that the string does not Fermi-Walker transport. Examples of solutions to the field equations are given and compared with solutions obtained from the postulated energy-momentum tensor of Letelier.
\end{abstract}

PACS numbers: $0440 \mathrm{~N}, 0570,9880 \mathrm{H}$

\section{Introduction to string fluid dynamics in general relativity}

The discussion of the cosmological implications of strings goes back nearly 20 years to the works of Zeldovich [1] and Vilenkin [2]. A string dust model was introduced by Letelier [3] from the description of the string function as a spacetime, surface-forming bivector

$$
\Sigma^{\mu \nu}=\epsilon^{A B} \frac{\partial x^{\mu}}{\partial \lambda^{A}} \frac{\partial x^{v}}{\partial \lambda^{B}}
$$

where

$$
\epsilon^{A B}= \begin{cases}1 & \text { if } A=0, B=1 \\ -1 & \text { if } A=1, B=0 \\ 0 & \text { if } A=B .\end{cases}
$$

The bivector string model was also discussed by Stachel [4].

In a previous work on the dynamics of a string fluid [5] it was noted that the form of equation (1) closely follows the form of the spin bivector developed by Halbwachs [6], and therefore the description of a string fluid energy density will use the parallel description of the velocity matrix used in discussions of fluid dynamics in continuum mechanics (see, for example, the discussions of fluids with spin and twist in metric affine geometry for a more complete discussion [7]). This means that in a fluid context, a Ray-Hilbert variational principle [8] can be developed for a string fluid in general relativity (GR) by introducing the string in terms of a set of tetrads

$$
\Sigma^{i j}=\rho \lambda(x)\left(a^{4 i} a^{3 j}-a^{4 j} a^{3 i}\right) \doteq \rho \sigma^{i j}
$$

where $\rho$ is the string density, $a^{\mu i}$ are the tetrads $\dagger$, where the Latin indices, $i=0,1,2,3$, are the holonomic coordinates, the Greek indices, $\mu=1,2,3,4$, are the anholonomic

$\dagger$ For a brief discussion of the possible choices for the tetrads chosen in equation (3), see the last section of the appendix. 
coordinates, and where $\lambda(x)$ are string (module) functions, respectively (considered as a parametric function), which will not be varied directly. For convenience, in the applications that follow in the penultimate section of this paper, the normalization for the string will be incorporated into a vector called the string vector $S_{j}$. The holonomic metric has signature $g_{i j}=(-1,1,1,1)$ and the anholonomic metric, $\eta_{\mu \nu}=(1,1,1,-1)$. We also identify the tetrad $a^{4 i} \equiv u^{i}$. Using equation (3), the equivalent 'angular' velocity matrix takes the form

$$
\omega^{i j}=\dot{a}^{\alpha i} a_{\alpha}{ }^{j}
$$

so that the string energy density is given by

$$
T_{S T}=\frac{1}{2} \Sigma^{i j} \omega_{i j}=\rho \lambda a^{4}{ }_{i} \dot{a}^{3 i} .
$$

Some additional justification for this string formulation is given by Nieto [9]. It is interesting to note the similarity of the string energy density in the fluid framework to that which occurs in general spin formulations [10].

In the previous work [5], the generality of the spacetime manifold was not restricted to the Riemannian form. However, this work will restrict itself to the Riemannian manifold since the examples discussed here will be compared to the behaviour of strings already formulated in general relativity (GR).

In the next section, the treatments of string fluids using both the improved and unimproved thermodynamic versions is described. In section 3, the energy-momentum tensors for the string fluid are developed. Examples of string solutions are given in section 4 and the conclusions and discussions are given in the last section.

\section{String fluid Lagrangian in general relativity}

The Ray-Hilbert Lagrangian for a string fluid in GR becomes

$$
\mathcal{L}_{\mathcal{G}}=\mathcal{L}_{\mathcal{M}}+\mathcal{L}_{\mathcal{G}}
$$

where $\mathcal{L}_{\mathcal{M}}$ is the matter Lagrangian

$$
\begin{gathered}
\mathcal{L}_{\mathcal{M}}=e\left\{-\rho[1+\epsilon(\rho, s)]+\lambda_{\mu \nu}\left(a^{\mu i} a^{\nu j} g_{i j}-\eta^{\mu \nu}\right)+\lambda_{2} \nabla_{i}\left(\rho u^{i}\right)+\lambda_{3} u^{i} X_{, i}\right. \\
\left.+\lambda_{4} u^{i} s_{, i}-\rho \lambda a^{4}{ }_{i} \dot{a}^{3 i}\right\}
\end{gathered}
$$

and $\mathcal{L}_{\mathcal{G}}$ is the gravitational field Lagrangian

$$
\mathcal{L}_{\mathcal{G}}=\frac{e R}{2 \kappa}
$$

where $e=\sqrt{-g}, \kappa=8 \pi G, R$ is the Riemann scalar in GR spacetime, $\lambda_{\mu \nu}$ are the Lagrange multipliers and we write $\lambda_{44}=\lambda_{1}$ in the variation. Note that only the third and fourth components of the tetrads occur in the Lagrangian (7) and are therefore the only active tetrads in the variational equations. The first and second components will therefore be suppressed in what follows. In the first part of this section, the string variables are not included in the thermodynamic formulations similar to the non-improved energy-momentum of spinning fluids [12]. In the following abbreviated subsection, the string variables are included in the extended thermodynamic formulations as in the improved energy-momentum of spinning fluids [11]. 


\section{Development of the energy-momentum tensor}

\subsection{Standard thermodynamics}

The standard thermodynamics for a perfect fluid for the Ray-Hilbert [8] variational principle is given by

$$
\mathrm{d} \epsilon=T \mathrm{~d} s+\frac{p}{\rho^{2}} \mathrm{~d} \rho
$$

The variational variables are then $g_{i j}, \rho, s, X, a^{\alpha i}$ and the various Lagrange multipliers.

3.1.1. Variational equations. (i) Tetrad and density variational equations. The variational equations become:

Density variation $\delta \rho$ :

$$
-\left(1+\epsilon+\frac{p}{\rho}\right)-\lambda_{2}-\lambda u^{i} \dot{a}^{3}{ }_{i}=0 .
$$

4-velocity $\delta u$ :

$-\rho \nabla_{i} \lambda_{2}+\lambda_{3} X_{, i}+\lambda_{4} s_{, i}+2 \lambda_{1} u_{i}+2 \lambda_{43} a^{3}{ }_{i}-\rho \lambda \dot{a}^{3}{ }_{i}-\rho \lambda u^{k} \nabla_{i} a_{k}^{3}=0$.

Tetrad $\delta a^{3 i}$ :

$$
2 \lambda_{33} a^{3}{ }_{i}+2 \lambda_{43} u_{i}+\rho u^{k} \nabla_{k}\left(\lambda u_{i}\right)=0 .
$$

The tetrad and density variations reveal the following relevant results for the Lagrange multipliers which will be useful in the analysis of the metric field equations and the transport properties of the string:

$$
\begin{aligned}
& 2 \lambda_{1}=[\rho(1+\epsilon)+p]-T_{S T} \\
& -\rho \dot{\lambda}_{2}=[\rho(1+\epsilon)+p]+T_{S T} \\
& 2 \lambda_{43}=-\rho \dot{\lambda} \\
& \lambda_{33} a^{3 i} a^{3 j}+\lambda_{43} u^{(i} a^{3 j)}+\frac{1}{2} \rho u^{k} \nabla_{k}\left[\lambda u^{(i}\right] a^{3 j)}=0 \\
& \lambda_{43} u^{(i} a^{3 j)}=-\frac{1}{2} \rho \dot{\lambda} u^{(i} a^{3 j)} .
\end{aligned}
$$

Adding equations (16) and (17) gives the useful result necessary to resolve the metric field equation

$$
\lambda_{33} a^{3 i} a^{3 j}+2 \lambda_{43} u^{(i} a^{3 j)}=-\frac{1}{2} \rho u^{k} \nabla_{k}\left[\lambda u^{(i}\right] a^{3 j)}-\frac{1}{2} \rho \dot{\lambda} u^{(i} a^{3 j)} .
$$

The final identity in this section comes from the antisymmetric combination of the tetrad variational equations which leads to a result similar to equation (16),

$$
\rho \lambda \dot{u}^{[i} a^{3 j]}=0 \text {. }
$$

This equation also has the interesting consequence that $\dot{u}^{i} \propto a^{3 i}$. In addition, we note the obvious identity

$$
\rho \lambda \dot{u}^{[i} a^{3 j]}=u_{k} \dot{u}^{[i} \Sigma^{j] k}
$$

(ii) Metric field equation. The variation with respect to the metric $g_{i j}$ gives the metric field equation

$$
\begin{aligned}
&-\frac{1}{2 \kappa} G^{i j}-\frac{1}{2} \rho(1+\epsilon) g^{i j}-\frac{1}{2} \dot{\lambda}_{2} g^{i j}+\lambda_{33} a^{3 i} a^{3 j}+2 \lambda_{43} u^{(i} a^{3 j)}+\lambda_{1} u^{i} u^{j} \\
&-\frac{1}{2} \rho \lambda u^{k} \dot{a}^{3}{ }_{k} g^{i j}-\rho \lambda u^{(i} \dot{a}^{3 j)}+\frac{1}{2} \nabla_{k}\left[u^{(i} \Sigma^{j) k}\right]+\frac{1}{2} u^{k} \nabla_{k}\left[\lambda u^{(i} a^{3 j)}\right]=0 .
\end{aligned}
$$


Inserting equations (13), (14) and (18) into equation (21) gives after rearranging

$$
\begin{aligned}
\frac{1}{2 \kappa} G^{i j}=\frac{1}{2}\{[ & {\left.[(1+\epsilon)+p] u^{i} u^{j}+p g^{i j}\right\}+\frac{1}{2} \rho u^{k} \nabla_{k}\left[\lambda u^{(i} a^{3 j)}\right] } \\
& -\frac{1}{2} \rho \dot{\lambda} u^{(i} a^{3 j)}-\frac{1}{2} T_{S T} u^{i} u^{j}+\frac{1}{2} \nabla_{k}\left[u^{(i} \Sigma^{j) k}\right]+\frac{1}{2} \rho u^{k} \nabla_{k}\left[\lambda u^{(i} a^{3 j)}\right] .
\end{aligned}
$$

All the ' $\lambda$ ' terms in equation (22) can be shown to satisfy the relationship

$$
\begin{gathered}
-\frac{1}{2} \rho u^{k} \nabla_{k}\left[\lambda u^{(i}\right] a^{3 j)}-\frac{1}{2} \rho \dot{\lambda} u^{(i} a^{3 j)}-\rho \lambda u^{(1 i} \dot{a}^{3 j)}+\frac{1}{2} \rho u^{k} \nabla_{k}\left[\lambda u^{(i} a^{3 j)}\right] \\
=-\frac{1}{2} u^{(i} \Sigma^{j) k} \dot{u}_{k}+\frac{1}{2} \nabla_{\ell}\left[u^{\ell} \Sigma^{k(j}\right] u^{i)} u_{k} .
\end{gathered}
$$

At first it would appear that the string energy density term $-\frac{1}{2} T_{S T} u^{i} u^{i}$ in equation (22) would renormalize the fluid energy. However, it will be shown below that this is not the case because of the identity

$$
T_{S T} u^{i} u^{j}=-u^{(i} \Sigma^{j) k} \dot{u}_{k} .
$$

Therefore substituting equations (23) and (24) into equation (22) gives the final results for the metric field equation

$$
G^{i j}=\kappa T_{S F}^{i j}
$$

where the string fluid energy-momentum tensor is given by

$$
T_{S F}^{i j}=T_{F}^{i j}+T_{S T}^{i j}
$$

where the perfect fluid energy-momentum tensor is

$$
T_{F}^{i j}=[\rho(1+\epsilon)+p] u^{i} u^{i}+p g^{i j}
$$

and

$$
T_{S T}^{i j}=\nabla_{k}\left[u^{(i} \Sigma^{j) k}\right]+\nabla_{\ell}\left[u^{\ell} \Sigma^{k(j}\right] u^{i)} u_{k}
$$

is the string energy-momentum tensor. Note that the combination of perfect fluid and string energy-momentum tensors given by equations (27) and (28) are the same form for a string fluid found by Smalley and Krisch [5] but for a string fluid in Riemann-Cartan spacetime. In reducing the metric equation to its final form given by equations (25)-(28), we have used the variational equations for the Lagrange multiplier for the fluid continuity and the 4 -velocity to obtain the relation equations (13) and (14)

$$
2 \lambda_{1}=[\rho(1+\epsilon)+p]-T_{S T}
$$

and

$$
\rho \dot{\lambda}_{2}=-[\rho(1+\epsilon)+p]-T_{S T}
$$

respectively. As a result of the extra factor $T_{S T}$ in equation (29), it is quite remarkable that the string energy density $T_{S T}$ does not occur in the fluid energy-momentum tensor given by equation (27). This renormalization does not occur because of the identity given by equation (24) in which the second term, which would have occurred in the string energymomentum tensor, cancels with the left-hand term, which would have occurred in the fluid energy-momentum tensor.

In the next subsection, the energy-momentum tensor is developed for the case where the energy is considered as a function of the string variables. 


\subsection{Extended thermodynamics}

Including the spin [12] and the shear momenta (or twist) [7] as thermodynamical variables has been discussed in earlier works. However, in this section the string variable is also treated as a thermodynamic variable. The energy now becomes

$$
\mathrm{d} \epsilon=T \mathrm{~d} s+\frac{p}{\rho^{2}} \mathrm{~d} \rho+\frac{1}{2} \omega_{i j} \mathrm{~d} \sigma^{i j} .
$$

Since most of the details are repetitions of the previous case, only the salient issues are presented for simplicity.

3.2.1. Variational equations. Even though the tetrad variations are slightly altered by the extended thermodynamics, the spinning string has the same Fermi-Walker transport properties described in the previous discussions based upon the standard thermodynamics. The changes in the metric field equation are given in the next section.

(i) Metric field equation. The metric field equation for the extended thermodynamics now becomes

$$
G^{i j}=\kappa T_{S F}^{i j}
$$

where the improved $T_{S F}^{i j}$ is now the sum of a perfect fluid energy-momentum tensor

$$
T_{F}^{i j}=[\rho(1+\epsilon)+p] u^{i} u^{i}+p g^{i j}
$$

(which is the same as before), and the string energy-momentum tensor is

$$
T_{S T}^{i j}=u^{(i} \Sigma^{j) k} \dot{u}_{k}-\dot{u}^{(i} \Sigma^{j) k} u_{k}+\nabla_{k}\left[u^{(i} \Sigma^{j) k}\right]+\nabla_{\ell}\left[u^{\ell} \Sigma^{k(j}\right] u^{i)} u_{k} .
$$

Before leaving the discussion of the spinning string fluid energy-momentum tensor, an interesting relationship for the temporal development of the string module function can be derived from the 4-momentum variational equation by taking the inner product with the tetrad $a^{3 i}$ to give

$-\rho a^{3 i} \nabla_{i} \lambda_{2}+\lambda_{3} a^{3 i} X_{, i}+\lambda_{4} a^{3 i} s_{, i}-\rho \dot{\lambda}-\rho \lambda u_{k} a^{3 i} \nabla_{i} a^{3 k}-\rho \lambda a^{3 i} \omega_{i j} a^{3 j}=0$.

Since the last term vanishes, this means that this expression applies to both models treated here. Equation (35) can be rewritten in a more illuminating form by defining the quantities $\lambda_{3}=\rho \hat{\lambda}_{3}, \lambda_{4}=\rho \hat{\lambda}_{4}$ and the spatial gradient along the string $a^{3 i} \nabla_{i} \mu \doteq \dot{\mu}$ so that

$$
\dot{\lambda}+\lambda u_{k} \dot{a}^{3 k}=\dot{\lambda}_{2}+\hat{\lambda}_{3} \dot{X}+\hat{\lambda}_{4} \dot{s}
$$

which shows explicitly the strong dependence of the time development of the string module function on the gradients of the matter along the string.

\section{Application to strings in general relativity}

The above discussion describes a universe populated by a string fluid (in contrast to the dust model described by Letelier [3,13]). However, to understand the content of this paper, it is necessary to briefly discuss the dust model as a basis for extending the concept to string fluid systems. This is described in the application below which extends a static, spherically symmetric, bivector string array in general relativity. 


\subsection{Application: extended dust model}

4.1.1. The stress-energy tensor. The stress-energy tensors discussed in the first part of the paper will be applied to finding exact solutions for a static, spherically symmetric bivector string array in GR. These solutions will be compared to the string system which have been studied by Letelier [3, 13] and Soleng [14]. The starting point for their calculation is the hypothesized string stress-energy tensor

$$
T_{i j}=\Sigma_{i}^{a} \Sigma_{j a}
$$

where for simplicity, a density and normalization factor have been absorbed into the definition of the string bivector which has now been defined in terms of the string vector $S_{j}$ discussed earlier after equation (3)

$$
\Sigma_{j a}=\frac{1}{4}\left(S_{j} u_{a}-S_{a} u_{j}\right)
$$

so that

$$
S^{j}=-4 \rho \lambda(x) a^{3 j} .
$$

In the previous sections, the stress-energy tensors given by equations (28) and (34) have been developed from dynamical arguments through a variational principle. These energymomentum tensors will be used for comparisons and extensions of the models of Letelier. Also the parameter $b$ will describe whether or not the string density has been included as a thermodynamic variable.

$$
\begin{aligned}
& b=1, \text { the string density is not a thermodynamic variable } \\
& b=2 \text {, the string density is a thermodynamic variable. }
\end{aligned}
$$

If in addition, the fluid is considered to be an anisotropic fluid with particle density $\rho$ and anisotropic stresses $t_{(a b)}$ where the indices $a, b=1,2,3$, then equations (28) and (34) can be written in the compact form

$$
\begin{array}{r}
T_{i j}=u_{i} u_{j}\left(\rho-\frac{1}{4}\left[S^{k}{ }_{; k}+b S^{k} \dot{u}_{k}\right]\right)+\frac{1}{8} b\left(\dot{u}_{i} S_{j}+\dot{u}_{j} S_{i}\right)+\frac{1}{4}\left(u_{i} S_{j}+u_{j} S_{i}\right) u_{; k}^{k} \\
+\frac{1}{4}\left(u_{i} \dot{S}_{j}+u_{j} \dot{S}_{i}\right)-\frac{1}{4} S^{k}\left(u_{i ; k} u_{j}+u_{j ; k} u_{i}\right)+t_{(a b)} A^{(a)}{ }_{i} A^{(b)}{ }_{j}
\end{array}
$$

where the $A^{(a)}{ }_{i}$ are a spacelike tetrad component of a set of tetrads (see the appendix) that will be used to diagonalize the metric and put the field equations into a more manageable form for calculational purposes. Also the components of this stress tensor are tabulated in the appendix for convenience. In what follows, this stress-energy content will be applied to two spacetimes: a modified Schwarzschild spacetime and the Friedman-Robertson-Walker (FRW) cosmological solution.

4.1.2. Modified Schwarzschild spacetime. The metric that will be considered for this application is given by

$$
\mathrm{d} s^{2}=-f \mathrm{~d} t^{2}+\mathrm{e}^{\mu} \mathrm{d} r^{2}+r^{2} \mathrm{~d} \theta^{2}+r^{2} \sin ^{2} \theta \mathrm{d} \varphi^{2}
$$

where $f$ and $\mu$ are functions of $r$ only. The set of tetrads that diagonalizes the metric is

$$
\begin{aligned}
& -u_{i}=A_{i}^{(0)}=(\sqrt{f}, 0,0,0) \\
& A^{(1)}{ }_{i}=\left(0, \mathrm{e}^{\mu / 2}, 0,0\right) \\
& A^{(2)}{ }_{i}=(0,0, r, 0) \\
& A^{(3)}{ }_{i}=(0,0,0, r \sin \theta) .
\end{aligned}
$$


The tetrad indexed radial and time Einstein tensors are

$$
\begin{aligned}
& G_{(r r)}=\mathrm{e}^{-\mu}\left(\frac{f_{, r}}{r f}+\frac{1}{r^{2}}\right)-\frac{1}{r^{2}} \\
& G_{(00)}=\frac{1}{r^{2}}+\mathrm{e}^{-\mu}\left(\frac{\mu_{r}}{r}-\frac{1}{r^{2}}\right) .
\end{aligned}
$$

If it is required that $G_{(r r)}=-G_{(00)}$, then

$$
\mathrm{e}^{\mu}=\frac{f_{0}}{f}
$$

where $f_{0}$ is a constant. With this requirement, then the complete tetrad indexed non-zero Einstein tensors become

$$
\begin{aligned}
& G_{(r r)}=-G_{(00)}=\frac{f_{, r}}{r f_{0}}+\frac{1}{r^{2}}\left(\frac{f}{f_{0}}-1\right) \\
& G_{(\theta \theta)}=G_{(\varphi \varphi)}=\frac{f_{, r, r}}{2 f_{0}}+\frac{f_{, r}}{r f_{0}} .
\end{aligned}
$$

From the appendix, the off-diagonal stress-energy content requires only a non-zero radial component for the string vector $S$

$$
\boldsymbol{S}=\left(0, S_{r}, 0,0\right)
$$

and therefore the stress-energy contribution becomes

$$
\begin{aligned}
& T_{(00)}=\rho-\left[S_{; k}^{k}+\frac{b-2}{2} f_{, r} S_{r}\right] \\
& \kappa T_{(11)}=t_{(11)}+\frac{1}{8} b f_{, r} S_{r} \\
& \kappa T_{(22)}=t_{(22)} \\
& \kappa T_{(33)}=t_{(33)}
\end{aligned}
$$

where $f_{0}$ is taken to be 1 . If it is assumed that $t_{(22)}=t_{(33)}=0$, then the modified Schwarzschild solution of Letelier [3, 13] is obtained

$$
f=1-a-\frac{2 M}{r}
$$

where $|a|<1$. With this solution, the field equations to be solved are

$$
-\frac{a}{r^{2}}=t_{(11)}+\frac{b}{8} f_{, r} S_{r}
$$

and

$$
\rho+t_{(11)}=\frac{1}{4}\left[f S_{r, r}+\frac{2 f S_{r}}{r}\right] .
$$

Note that the equation of state equation (56) is independent of the choice of the thermodynamic variables. This type of a global monopole has been discussed by Barriola and Vilenkin [15]. 


\section{(i) Two Schwarzschild spacetime models}

(a) Dust model. In this model, $t_{(11)}=0$. Then using equation (55), the string vector is seen to be the constant

$$
S_{r}=-\frac{4 a}{b M}
$$

and the fluid density for this model becomes

$$
\rho=-\frac{2 a f}{b M r} \text {. }
$$

The requirement that $a$ should be negative to get positive densities means that the event horizon will be contracted by the presence of the constant string field.

(b) Equation of state model. In this model, the equation of the string system is given by $t_{(11)}=-\rho$. In this case, the string vector follows directly from the equation of state (equation (56)) so that

$$
S_{r}=\frac{S_{0}}{r^{2}}
$$

where $S_{0}$ is an integration constant called the string parameter. Thus the stress and fluid density are given by

$$
-t_{(11)}=\rho=\frac{a}{r^{2}}+\frac{b M S_{0}}{4 r^{2}} .
$$

Thus all fluids are singular on the horizon. For large $r$, the fluid has the very simple behaviour of order $\vartheta\left(1 / r^{2}\right)$

$$
-t_{(11)}=\rho \approx \frac{a}{r^{2}} .
$$

In this model, the string parameter $S_{0}$ is not directly linked to the parameter $a$. Letelier's string cloud model [3] is most similar to the large-distance behaviour of the second case in which he finds the string cloud falling off as $r^{-2}$. The constant string vector function is new. Letelier [16] has discussed a model with stress but of the Schwarzschild form.

(ii) A cosmological model

The second example is an isotropic spherically symmetric cosmology described by the metric

$$
\mathrm{d} s^{2}=-\mathrm{d} t^{2}+a^{2}(t)\left[\frac{\mathrm{d} r^{2}}{1-k r^{2}}+r^{2} \mathrm{~d} \theta^{2}+r^{2} \sin ^{2} \theta \mathrm{d} \varphi^{2}\right] .
$$

The diagonal field equations for this system are now given by

$$
\rho-\frac{1}{4} S_{; k}^{k}+3 p=-\frac{6}{a} \frac{\mathrm{d}^{2} a}{\mathrm{~d} t^{2}}
$$

and

$$
\rho-\frac{1}{4} S_{; k}^{k}=\frac{3}{a^{2}}\left(\frac{\mathrm{d} a}{\mathrm{~d} t}\right)^{2}+\frac{3 k}{a^{2}} .
$$

These field equations are identical to the usual FRW field equations with the replacement of the density by the density minus the string function divergence. Upon specializing to dust, as in the stringless cosmology, it can be shown that

$$
\rho-\frac{1}{4} S_{; k}^{k}=\frac{\rho_{0}}{a^{3}}
$$


with $\rho_{0}$ a constant. The time behaviour of the string functions follows from the off-diagonal field equations that are given, using the appendix, as

$$
S_{i}=S_{i}(r, \theta) a^{-3}(t)
$$

where $i$ is given by the spatial components $r, \theta, \varphi$. Equation (64), when applied to the dust stringless cosmology, implies that the universe cannot be static if $k=0$ or -1 . When strings are included this is no longer true. It is possible to have the radius $a$ constant for $k=0$ if

$$
\rho=\frac{1}{4} S_{; k}^{k} .
$$

For $k=+1$, there are some differences in the critical density values for the expansion to cease and reconstriction to start. In the stringless cosmology, the critical radius for the turning point is

$$
a_{c}=\frac{3}{\rho} .
$$

The actual mass density can be greater than the stringless density if the string function is positive and less than the stringless density if the string function is negative.

\section{Conclusions and discussions}

We have developed a stress-energy tensor describing a string fluid. The fluid potentially can have mass and stress content. The stress tensor is valid in general relativity for the string variable being either treated as a thermodynamic variable or not. Two applications were considered.

A modified Schwarzschild spacetime was found which was similar to that of Letelier [3] in which the presence of the strings moved the horizon. In the dust solution, the requirement of positive string density implied that the horizon was larger that its Schwarzschild value due to the presence of the string system. The dust solution presented here has a constant string density and a mass density falling off like $r^{-1}$. The second Schwarzschild model has string tension and the same $r^{-2}$ drop off in density as Letelier [17]. For this model, the position of the horizon was larger than its Schwarzschild value. The choice of the thermodynamic behaviour produced only factors of two difference in the numerical results for these models.

The second application considered here was a homogeneous, isotropic cosmology. In this example, the presence of the string system produced some interesting modifications to the usual stringless perfect fluid cosmology. In addition to the usual expanding solutions, for a particular value of the density, a static solution is possible. The choice of the thermodynamic behaviour did not appear in this example. The difference in the thermodynamic behaviour is linked to the presence of acceleration in the spacetime. In contrast, the FRW metric is unaccelerated.

The stress-energy tensor presented in this paper has provided an enriched spectrum of solutions. The enlarged parameter content of this fluid makes this approach a valuable tool for modelling an expanded class of spacetimes. It is expected that the addition of spin to string models will also be of interest and it is suggested that such terms will have implications for the horizon as well. As yet, the thermodynamic parameter $b$ has not been a significant factor in the determination of the event horizon and it is not yet clear whether the addition of spin will change this conclusion in GR. These ideas are presently under investigation and will be reported elsewhere. 


\section{Appendix}

\section{A.1. Metric and tetrads}

The general spacetime metric is given by

$$
\mathrm{d} s^{2}=-f \mathrm{~d} t^{2}+\mathrm{e}^{\mu} \mathrm{d} r^{2}+\mathrm{e}^{\beta} \mathrm{d} \theta^{2}+\frac{D^{2}}{f} \mathrm{~d} \varphi^{2}
$$

where $f, \mu, \beta$ and $D$ are considered to be functions of $r$ and $\theta$. A convenient set of tetrads that diagonalize the metric is given by

$$
\begin{aligned}
-u_{i} & \equiv A^{(0)}{ }_{i}=(\sqrt{f}, 0,0,0) \\
A^{(1)}{ }_{i} & =\left(0, \mathrm{e}^{\mu / 2}, 0,0\right) \\
A^{(2)} & =\left(0,0, \mathrm{e}^{\beta / 2}, 0\right) \\
A^{(3)} & =(0,0,0, D / \sqrt{f}) .
\end{aligned}
$$

\section{A.2. Stress-energy components}

The string vector has the components

$$
S=\left(0, S_{r}, S_{\theta}, S_{\varphi}\right) .
$$

The tetrad indexed diagonal components of the stress-energy tensor are given by

$$
\begin{aligned}
& \kappa T_{(00)}=\rho-\frac{1}{4} S_{; a}^{a}+\frac{b-2}{2 f} f_{, a} S^{a} \\
& \kappa T_{(11)}=t_{(11)}-\frac{b}{8 f} f_{, r} S^{r} \\
& \kappa T_{(22)}=t_{(22)}+\frac{b}{8 f} f_{, \theta} S^{\theta} \\
& \kappa T_{(33)}=t_{(33)}
\end{aligned}
$$

where the summation index $a=r, \theta$. Define the parameter $A$ such that

$$
A=\frac{1}{2}(\dot{\mu}+\dot{\beta})+\frac{\dot{D}}{D}-\frac{\dot{f}}{2 f} .
$$

Then the off-diagonal stress-energy components are given by

$$
\begin{array}{lr}
\kappa T_{(r 0)}=-\frac{\mathrm{e}^{\mu / 2}}{4 \sqrt{f}}\left(S_{, 0}^{r}+A S^{r}\right) ; & \kappa T_{(r \theta)}=+b \frac{\mathrm{e}^{-(\mu+\beta) / 2}}{8 f} \\
f_{,(r} S_{\theta)} \kappa T_{\theta(0)}=-\frac{\mathrm{e}^{\beta / 2}}{4 \sqrt{f}}\left(S_{, 0}^{\theta}+A S^{\theta}\right) ; & \kappa T_{(r \varphi)}=+b \frac{\mathrm{e}^{-\mu / 2}}{16 D f} f_{, r} S_{\varphi} \\
\kappa T_{(0 \varphi)}=-\frac{\mathrm{e}^{\mu / 2}}{4 \sqrt{f}}\left(S_{, 0}^{\varphi}+A S^{\varphi}\right) ; & \kappa T_{(\theta \varphi)}=+b \frac{\mathrm{e}^{-\mu / 2}}{16 D f} f_{, \theta} S_{\varphi} .
\end{array}
$$

\section{A.3. Tetrad choice}

The choice of tetrads is a free choice for calculations using the Ray-Hilbert variational principle [8] for a broad class of problems. The choice of tetrads enters into the calculation 
through equation (4) in finding the spin angular velocity. Writing equation (4) explicitly in its implicit antisymmetric form

$$
\omega^{i j}=\frac{1}{2}\left(\dot{a}^{\alpha i} a_{\alpha}{ }^{j}-\dot{a}^{\alpha j} a_{\alpha}{ }^{i}\right)
$$

and expanding gives

$$
2 \sqrt{f} \omega^{i j}=a_{\alpha}^{j} \partial_{0} a^{i}-a_{\alpha}{ }^{i} \partial_{0} a^{j}+\Gamma^{i}{ }_{0 k} g^{j k}-\Gamma^{j}{ }_{0 k} g^{i k} .
$$

If there is no time dependence in the metric, the angular velocity clearly does not depend on the tetrad choice. If the time dependence in the metric is an overall factor, the time derivatives cancel. If the metric has the unaccelerated cosmological form with a conformal time dependence on the spatial part of the metric, again the derivatives cancel, and there is no dependence on the choice of the tetrads. The examples in this paper all fall within these very broad classes.

\section{References}

[1] Zeldovich Ya B 1980 Mon. Not. R. Astron. Soc. 192663

[2] Vilenkin A 1981 Phys. Rev. Lett. 461169

Vilenkin A 1981 Phys. Rev. Lett. 461496 (erratum)

[3] Letelier P S 1979 Phys. Rev. D 201294

[4] Stachel J 1980 Phys. Rev. D 212171

[5] Smalley L L and Krisch J P 1996 Class. Quantum Grav. 13 L19

[6] Halbwachs F 1960 Theorie Relativiste des Fluide a Spin (Paris: Gauthier-Villar)

[7] Smalley L L and Krisch J P 1995 J. Math. Phys. 36778

[8] Ray J R 1972 J. Math. Phys. 131451

[9] Nieto J A 1995 From a relativistic point particle to string theory Preprint Department of Physics and Mathematics, University of Michoacana, Mexico

[10] Ray J R and Smalley L L 1982 Phys. Rev. D 262619

[11] Ray J R, Smalley L L and Krisch J P 1987 Phys. Rev. D 353261

[12] Ray J R and Smalley L L 1982 Phys. Rev. Lett. 491059

Ray J R and Smalley L L 1982 Phys. Rev. Lett. 50525 (erratum)

[13] Letelier P S 1983 Phys. Rev. D 282414

[14] Soleng H H 1995 Gen. Rel. Grav. 27367

[15] Barriola M and Vilenkin A 1980 Phys. Rev. Lett. 63341

[16] Letelier P S 1981 Nuovo Cimento B 63519

[17] Letelier P S 1980 Phys. Rev. D 22807 